\title{
LA RURALIDAD EN UN MUSEO. TENSIONES EN LA MUSEALIZACIÓN DE CUCAITA, BOYACÁ, COLOMBIA*
}

\section{CARLOS ANDRÉS CARREÑO HERNÁNDEZ**}

Recibido: 21 de junio de 2017

Aprobado: 18 de agosto de 2017

Artículo de Investigación

\footnotetext{
* Artículo derivado de la investigación titulada: "Narrativa y territorio. Tensiones en la musealización de Cucaita", adelantada por el autor, para optar al título de Magíster en Patrimonio Cultural, de la Universidad Pedagógica y Tecnológica de Colombia. Investigación financiada por la Dirección de Investigaciones de la misma universidad.

** Profesional en Conservación y Restauración de Bienes Muebles. Magíster en Patrimonio Cultural Universidad Pedagógica y Tecnológica de Colombia). Investigador del Grupo de investigaciones arqueológicas e históricas y de la Fundación Piedra Alta.Colombia.E-mail: conservacion.restauracion76@

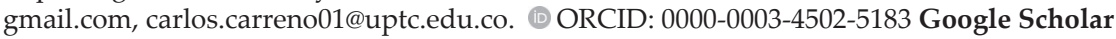




\title{
Resumen
}

Objetivo. Reflexionar en torno a un proceso de investigación, adelantado por el autor entre los años 2015 y 2016, para la optimización de un museo en el contexto regional colombiano, específicamente en el municipio de Cucaita, departamento de Boyacá - Metodología. A través de una metodología participativa, las comunidades fueron parte integrante y actuante a lo largo del proceso. Resultados y conclusiones. Propuesta de un planteamiento museológico que, desde una perspectiva crítica, interprete los aportes que en su momento diera la denominada nueva museología aplicándolos a un contexto rural colombiano. El proceso permitió entender el campo de investigación de los museos como un espacio posible para el debate, en torno a su función, pertinencia y permanencia al interior de unas comunidades, que como las campesinas requieren de referentes que fortalezcan sus vínculos socioculturales, para reconocerse como colectividad consciente al interior de las dinámicas contemporáneas.

Palabras clave: museo, territorio, comunidad, nodo, desarrollo.

\section{RURALITY IN A MUSEUM. TENSIONS IN THE MUSEALIZATION OF CUCAITA, BOYACÁ, COLOMBIA}

\begin{abstract}
Objective: To reflect on a research process carried out between the years 2015 and 2016, for the optimization of a museum in the Colombian regional context, specifically in the municipality of Cucaita, Department of Boyaca. Methodology: Through a participating methodology, the communities were a comprehensive and acting part along the process. Results and conclusions: Proposal of a museological approach that, from a critical perspective, interprets the contributions that the socalled new museology provided, applying them to a rural context in Colombia. The process allowed the understanding of the museum investigation field as a possible space for debate around its function, relevance and permanence within communities which, like the farmers community, require referents that strengthen their sociocultural links to be recognized as conscious communities within contemporary dynamics.
\end{abstract}

Key words: museum, territory, community, node, development. 
La creación del Museo de Cucaita la pensamos como homenaje a las familias campesinas del municipio, para que las nuevas generaciones conozcan los objetos que hicieron parte de la cultura material de sus abuelos" (H. Neiza, comunicación personal, 15 de julio de 2015)

La puritica verdad a yo me da jartera entrar a ese museo de la alcaldía, a yo me parece que ese museo no sirve para nada, prefiero quedarme trabajando en mi parcela, esta huertica que me da pa las arepitas y la chicha. (M. Vanegas, comunicación personal, 12 de octubre de 2015)

\section{Introducción}

U no de los detonantes que incitó mi interés por estudiar el Museo de Cucaita ${ }^{1}$ ocurrió tras una visita guiada realizada con estudiantes de la sede bachillerato de la Institución

Educativa San Felipe, ubicada en el centro urbano del municipio, poco después de su reinauguración; luego del montaje museográfico diseñado por el curador bogotano Guillermo Vanegas Flórez, y producido por la Corporación Santa Clara la Real, en 2013, en homenaje a los ancestros de ésta región. Cuando estábamos comentando sobre algunos de los objetos exhibidos, un pequeño grupo no dejaba de reír, de esa manera que solo los jóvenes saben hacerlo para generar inquietud en los adultos que creen estar conversando de manera directa con ellos. Al preguntar la razón de tal risa, uno de ellos señaló uno de los yugos exhibidos en la pared sur del museo, y respondió: “...profe, es que no entiendo pa' qué sirve eso ahí colgado, eso sirve es sobre el buey pa' mover la tierra de mi abuelo Vicente..." (J.M. Niño, 14 años de edad). Ante una intervención tan corta pero contundente, intenté finalizar, de la manera más rápida y digna posible la actividad.

Este cuestionamiento, me llevó a reflexionar sobre la relevancia y pertinencia de este espacio. ¿Los objetos exhibidos son lo importante? ¿la capacidad narrativa del museo es usada en toda su dimensión? ¿es la función de un museo el limitarse a exhibir? Estas preguntas, que ya se habían hecho muchos antes, a partir de la década del 70, conllevaron a nivel internacional a la definición de nuevos conceptos que convertirían a los museos en espacios de interpretación y análisis, derivados de la nueva museología.

\footnotetext{
${ }^{1}$ Museo municipal del municipio de Cucaita, ubicado en la provincia Centro, departamento de Boyacá, Colombia. Fue creado por la alcaldía municipal, a través del investigador local Henry Neiza Rodríguez, en el año 2007,
} 


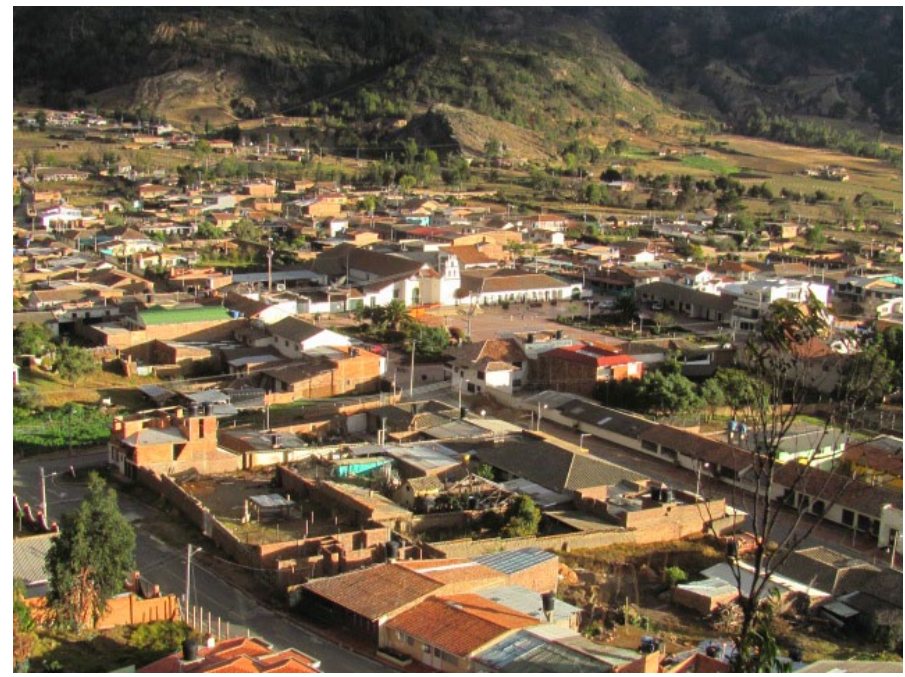

Figura 1: Vista aérea del municipio de Cucaita, Boyacá, Colombia.

Fuente: Carlos Carreño (2016).

\section{Un museo en un territorio:}

Uno de los resultados de mi experiencia, tras haber habitado e interpretado Cucaita, como lugar de coexistencia de comunidades y de ocurrencia de sus cotidianidades, es el entender que un territorio es una compleja red de sentidos, a través de la cual se interconectan una serie de nodos, que en diversos niveles, son elementos activos que dan vida y significado al lugar como espacio habitado.

Dichos nodos son entes activos en constante transformación, que se concretan a partir de la consolidación en el territorio de experiencias significativas de las comunidades, para intentar suplir necesidades productivas, espirituales y/o sociales. Ejemplos de estos nodos pueden ser las lógicas de ocupar el territorio, visibles en los asentamientos, el hogar, la parcela, o bien las relaciones de intercambio, que se dan en escuelas, el parque principal del centro urbano, el templo parroquial, o la plaza de mercado, entre otros.

Para que estos nodos existan como tal, y la red se configure, es necesario que los primeros contengan un ente cohesionador, a través del cual, (i) se interconecten los nodos entre sí, y (ii), se consolide la forma de cada nodo, ya que a modo de núcleo sostiene la gravitación de elementos que hacen parte de ciertos aspectos de las relaciones humanas locales. Estos entes son los referentes, que permiten que las personas se reconozcan frente a otras como parte de un mismo contexto sociocultural. 
A través de estos referentes no solo se consolida la red en el territorio, sino que su función de interconexión, brinda el dinamismo a dicha red, es decir, que estos referentes se conectan por medio de vasos comunicantes, cuya movilidad y flujo constante, hacen que los territorios sean espacios vivos, existentes, en movimiento.

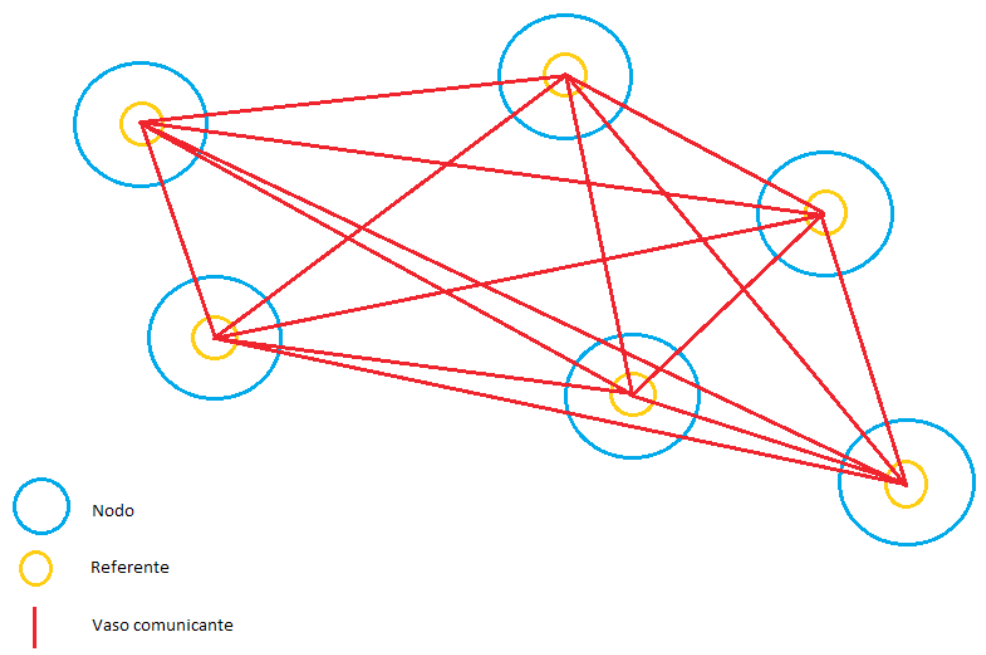

Gráfico 1. Corema de red territorial.

Fuente: Carlos Carreño (2016)

Esos referentes o núcleos obedecen a lógicas locales, construidas por las personas mismas y su cotidianidad como colectividad, a través de sus prácticas y necesidades, configurando de esta manera el corpus actual de dichos territorios, es decir, que el territorio es el resultado, siempre activo y propenso a la transformación, del devenir histórico y la trayectoria de las colectividades.

Entre esas lógicas están aquellas asociadas a las dinámicas económicas, sociales y culturales. En este sentido, como ya se mencionó, son producto del trasegar de las comunidades que apoyadas en sus creencias, aprendizajes, errores y aciertos, afianzan una forma de pensar y valorar su territorio y por este trasegar construyen una serie de estrategias para habitarlo y hacerlo propio. En palabras de Maurice Halbwachs (2002): "todos los movimientos de un grupo pueden traducirse en términos espaciales, y el lugar ocupado por este grupo no es más que la reunión de todos los términos" (p. 10). 


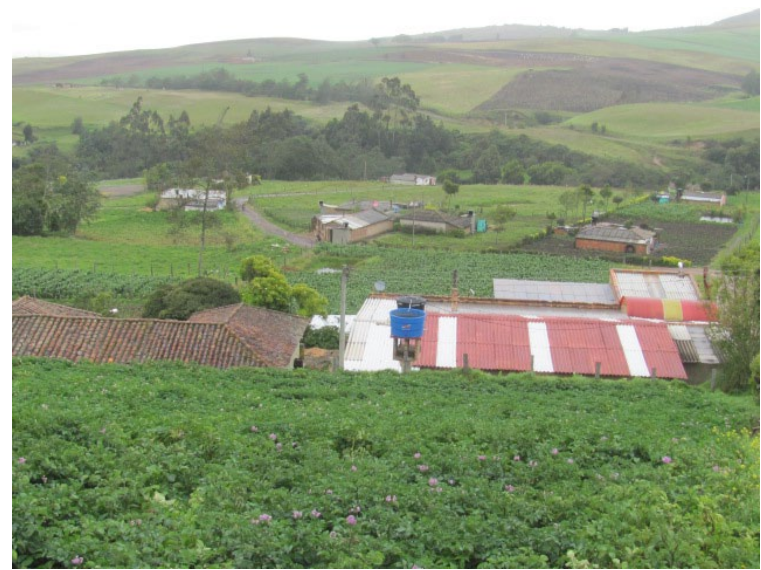

Figura 2. Parcelas en vereda Pijaos, municipio de Cucaita, Boyacá, Colombia Fuente: Carlos Carreño (2015)

En esta red se intentó insertar al Museo de Cucaita como referente, por parte de algunas personas que, siguiendo modelos exógenos, intentaron emular los museos de otros contextos, a través de la imposición de un espacio para exhibir objetos en medio de un contexto rural, de manera un tanto exotizante, y alejada de los procesos cotidianos del lugar. El resultado fue una serie de objetos enajenados de su realidad y actualidad, que como el yugo mencionado, se convirtieron en cosas estáticas, silenciando su papel como elemento dinamizador de la memoria, evidente en las casas y espacios campesinos.

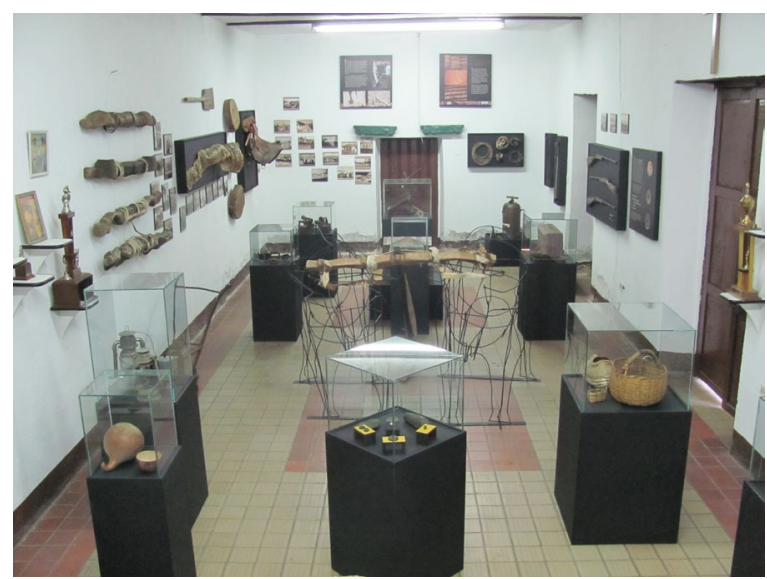

Figura 3. Vista interior del Museo de Cucaita.

Fuente: Carlos Carreño (2015) 
En este sentido, el interés se centra en cómo revitalizar dicho museo, para que se inserte de manera efectiva al interior de la cotidianidad del lugar, y de esta forma se pueda conectar con la red territorial, a modo de referente y, cuyo nodo se va construyendo en la medida en que las personas hagan uso del mismo de manera consciente y bajo sus necesidades reales.

Así pues, con esta investigación, planteo convertir al Museo de Cucaita en un dinamizador de sentido; en el referente de un nodo que exprese y haga conscientes las lógicas locales de las comunidades que habitan el territorio. Este proyecto busca la manera de abandonar definitivamente la concepción tradicional de museo, en donde la importancia de este espacio radica exclusivamente en la conservación de los valores de los objetos mismos, a través de los cuales se pretende construir un discurso univalente (De Carli, 2004), y no de los valores atribuidos por las comunidades, que al fin y al cabo son las que le otorgan sentido.

Pienso yo que eso del museo debería ser algo más llamativo, las cosas esas son bonitas, no digo que no, pero eso parece es el cuarto de chécheres viejos, de esos que las casas de los antiguos se usaban para guardar lo que ya no sirve. (J. Gómez, comunicación personal, 10 de octubre de 2015)

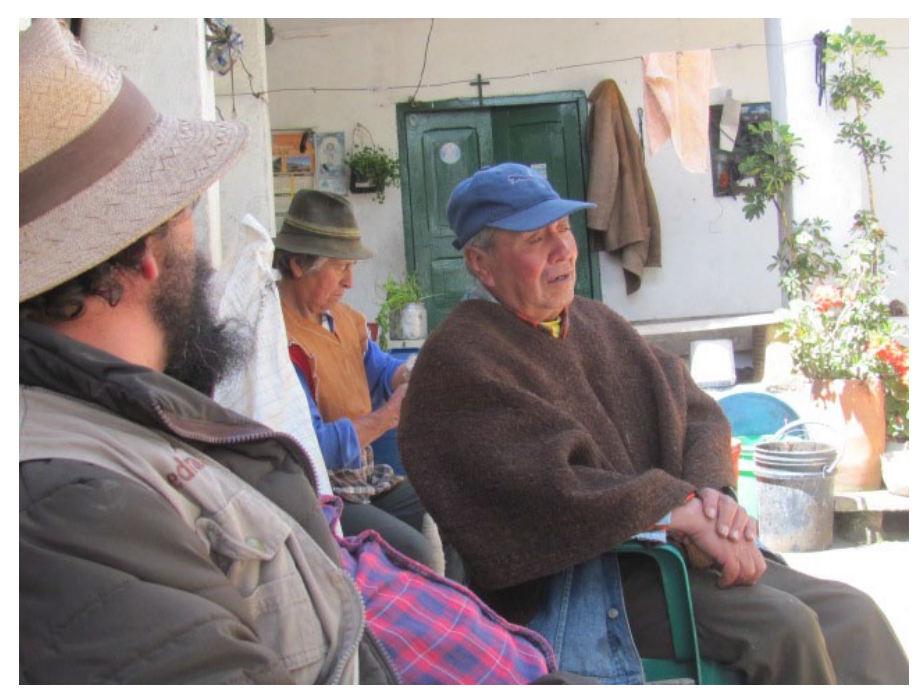

Figura 4. Conversación con José Gustavo Gómez y su esposa María Estrella López.

Vereda Lluviosos, municipio de Cucaita, Boyacá, Colombia.

Fuente: Carlos Carreño (2015) 
Estas apreciaciones sobre el museo, por parte de los miembros de las comunidades de Cucaita, lleva a preguntarse con Halbwachs (2002): “ ¿... cómo recrear corrientes de pensamientos colectivos que tomen su impulso del pasado cuando todo lo que permanece es el presente?..." (p. 8), y cómo se comporta el corpus del territorio, construido desde la oralidad de las personas.

Bajo la idea de nodo, y basado en un referente que está llamado a detonar una consciencia colectiva (Halbwachs, 2002), entendiendo esta consciencia colectiva como fuerza cohesionadora de las comunidades, considero la memoria de las mismas como parte activa de ese proceso constructor de territorio, y de metabolismo territorial "...creo que es porque la cultura apacigua los ánimos, nos vuelve amigos, conocidos, como que nos familiariza como seres humanos..." (A. Gil Tovar, comunicación personal, 13 de agosto de 2015).

En este escenario busco que el Museo de Cucaita atienda el "... llamado de alerta y crítica a los museos tradicionales, la exigencia de un cambio y la aceptación de un compromiso..." (De Carli, 2004, p. 58). Así, y según la Resolución de la Mesa Redonda ICOM-UNESCO

el museo es una institución al servicio de la sociedad de la cual es parte inalienable y tiene en su esencia misma los elementos que le permiten participar en la formación de la conciencia de las comunidades a las cuales sirve y a través de esta conciencia puede contribuir a llevar a la acción a dichas comunidades, proyectando su actividad en el ámbito histórico que debe rematar en la problemática actual; es decir anudando el pasado con el presente y comprometiéndose con los cambios estructurales imperante y provocando otros. (De Carli, 2004, p. 58)

Pensar de esta forma el Museo de Cucaita implica desmontar su papel como custodio de una cantidad de objetos antiguos, para entrar a reflexionar sobre cómo transmitir, alimentar y dinamizar el conocimiento de las comunidades; de pensar históricamente como ejercicio de análisis (Cohen, 2012) que "...la historia está hecha más de transformaciones que de supervivencias..." (Londoño, 1989, p. 95); de convertir al museo en un ejercicio dialectico de los “...porqués de esas transformaciones y así evitar el mero arrume etnográfico de permanencias" (Londoño, 1989, p. 95), y en un espacio en el que 
Los jóvenes nos expliquen a nosotros los viejos sus cosas, sus razones pa' comportarse como se comportan, para cambiar tuitico eso que los antiguos nos habían dejado ya. Es que las cosas ya no son lo mismo y nosotros los viejos tenemos que abrir bien las orejas para comunicarnos con los otros. (B. López, comunicación personal, 23 de noviembre de 2015)

Para este propósito, parto de la idea del museo como producto de colectividades, donde las narrativas que contenga contemplen las lógicas locales y contextos rurales de las comunidades cucaitenses. Ya que "lo importante es llegar a distinguir entre la singularidad de unos hechos y el verdadero alcance de unos mecanismos sociales, sin referencia a los cuales esos hechos resultan inexplicables" (Le Golf y Nora, 1978, p. 17).

Establecer la ruralidad como contexto, como narrativa y como campo de interacción del museo, es una forma de plantearlo como espacio cultural cuya prioridad son los habitantes y no una unidad productiva enfocada a visitantes foráneos (Vázquez, 2008). Este es otro de los retos de mi propuesta, puesto que la vocación, la función y el uso cambian con respecto a los museos a los cuales estamos acostumbrados en el contexto boyacense y colombiano. Estos museos han sido diseñados para ser visitados con una mirada un tanto omnisciente y poco, por no decir nunca, comprometida con la narrativa del contexto que observa. Pretendo que el museo responda a las lógicas locales de función y uso: "le metería un tractor jajajajaja para hacer una huerta o un sembrado de papa jajajajaj, un museo sembrado jajajaja" (A. Rodríguez, comunicación personal, 21 de septiembre de 2015).

Es en esa ruralidad que para el museo, como espacio cultural de carácter público, debo buscar nuevas formas de ser referente y activarlo en su contexto sociocultural, cumpliendo con una función real para el beneficio de la comunidad, o mejor aún, de las comunidades a las cuales se debe. Igualmente asumo que se trata de una ruralidad que no es estática, sino dinámica.

Eso es importante que se oiga en el pueblo, que nosotros los campesinos no somos unos muñequitos de mostrar, somos seres humanos, si señorito, somos gente que nos movemos, que cultivamos, que buscamos soluciones para nuestras necesidades. Es que a veces yo me pregunto si en realidad se valora lo bonito que tiene cada cultivo, nunca es igual, nunca es lo mismo, así como el agua de la quebrada que pasa po aquí...la tierra es una cosa viva mijito. (H. Otálora, comunicación personal, diciembre de 2015) 


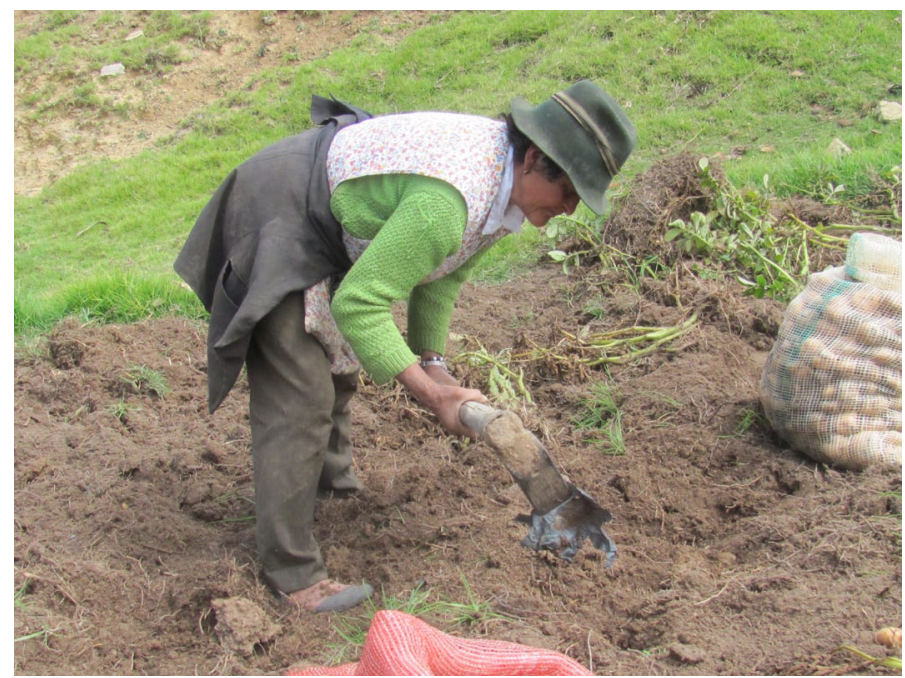

Figura 5. Helena Otálora López cosechando papa en su parcela.

Vereda Escalones, municipio de Cucaita, Boyacá, Colombia.

Fuente: Carlos Carreño (2015)

\section{Fragmentación del territorio}

El reconocimiento del territorio, de la ruralidad, lo hice de la mano de las comunidades, a través de los espacios de conversación, talleres y recorridos dialogados, en los que se buscó entender a Cucaita como espacio social. Durante la investigación, Cucaita se entendió como territorio “... producto de la experiencia, la percepción y la imaginación..." (Harvey, 1989, como se citó en Ortiz, 1998, p.114).

Esto me permitió observar que Cucaita, es una entidad territorial “... de inscripción de la cultura, marco o área de distribución de prácticas [...] como objeto de representación y apego afectivo y un símbolo de pertenencia socioterritorial..." (Ortiz, 1998, p.114) que se encuentra en proceso de fragmentación.

Algunos de los causantes externos de esta fragmentación se asocian al cambio climático y su incidencia en las economías domésticas, generando migraciones, los altos costos de los insumos y los bajos precios de los productos agrícolas en el mercado, haciendo del campo una economía insostenible; así como el desinterés y la falta de oportunidades entre las nuevas generaciones y su consecuente desplazamiento hacia ciudades como Tunja, Chiquinquirá y Bogotá, entre otros factores. Y esta fragmentación afecta esa relación de equilibrio que contiene el territorio, “...el lugar 
más allá de su dimensión física, como una experiencia vital que integra naturaleza, significados y relaciones sociales" (Sack, 1988, como se citó en Ortiz 1998, p. 114).
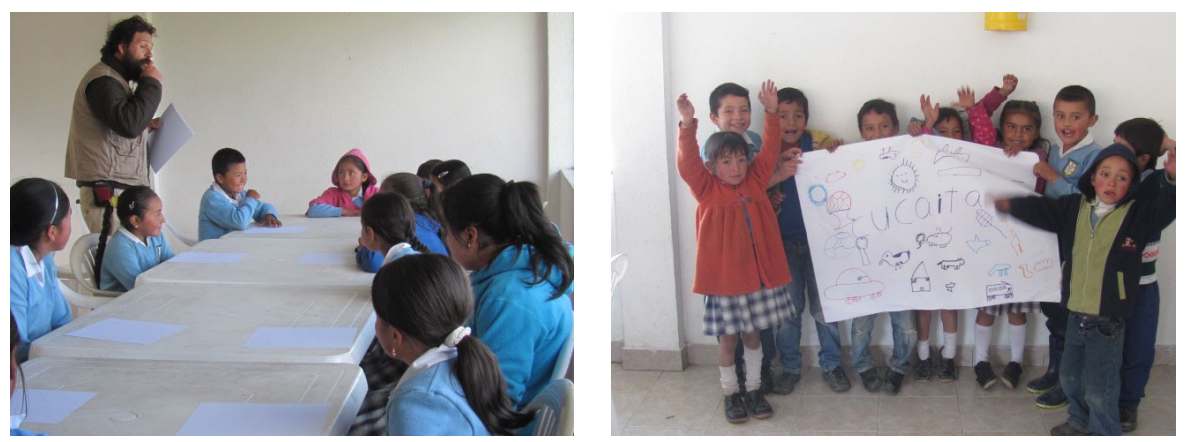

Figuras 6, 7. Momentos espacios de diálogo con niños de escuela rural vereda Escalones, Municipio de Cucaita, Boyacá, Colombia.

Fuente: Carlos Carreño (2015)

Siendo relevante para el proyecto del museo, como referente de un nodo de la red territorial, generar pensamientos colectivos que anude el pasado con el presente; esta fragmentación es un riesgo sobre el cual se plantea actuar. Una de las consecuencias de esta fragmentación, es el debilitamiento de la transmisión de los conocimientos, pues la estructura familiar también se ha debilitado y no hay comunicación intergeneracional.

Si hay un vacío intergeneracional dentro de una cultura de tradición oral, las posibilidades de reproducción de todo este capital cultural están amenazadas en la medida en que no habrá posibilidad de asegurar su continuidad a través de los procesos de socialización primaria al interior del hogar. (López, 1989, p. 147)

En consecuencia, la perdurabilidad en el tiempo del acervo sociocultural, entendido este como aquel conocimiento producto de la interacción en comunidad en sus lugares, es un tema de preocupación para los cucaitenses, que ven cómo sus hijos y nietos asumen actitudes vergonzantes hacia sus orígenes campesinos.

Ahí chinitico si viera busté la tristeza de eso, yo de mi parte tuve seis varones con el patrón (dirigiendo la mirada hacia Don José Gustavo Gómez quien estaba sentado a mi lado durante la conversación) y quen dijera, los chivatos se jueron pa la capital, alla hicieron sus vidas y pu' aquí regresan apenas pa saludar, pero eso pareciera vergüenza 
lo que los tuye, quesque ni citadinos que jueran...". (M. López, comunicación personal, 20 de noviembre de 2015, lo narra mientras dirige su mirada triste hacia un arrume de juguetes que se encuentran sobre una mesa en una esquina de su casa)

Ligado a lo anterior, encuentro que la educación formal es otro factor externo que incide en la fragmentación del territorio. Durante el trabajo de campo con la comunidad escolar observé la implementación de estrategias pedagógicas no pensadas para los contextos rurales, sino más propicias para los centros urbanos, donde se transmiten valores que distan de la realidad cotidiana de los niños y jóvenes que habitan el campo, por lo que “...la transmisión de estos valores va creando un verdadero conflicto entre la unidad doméstica y la escuela, para no hablar sino del problema de los ciclos agrarios y los calendarios escolares" (López, 1989, p. 148).

En efecto, esta afectación por parte de una política de educación nacional que pareciera ir en contravía con la condición rural de la mayoría de la población, ha incidido también en Boyacá “...uno de los departamentos con mayor tasa de deserción escolar por este enfrentamiento y por las contradicciones entre la escuela y la vida cotidiana del estudiante" (López 1989 p. 148),

Para mi caso yo me salí como en $3^{\text {o }}$ de bachillerato porque sentí de verdad que eso no me servía para nada, disque aprenderse las capitales de Europa, la fecha que nació Simón Bolívar y los logros de Napoleón. Con Marco Tulio siempre fregábamos en clase y preguntábamos, medio en broma medio en serio, porque en esas líneas del tiempo nunca se hablaba de cosas concretas de Cucaita". (R. Moreno, comunicación personal, 12 de diciembre de 2015)

Es, pues, en la escuela donde tienen inicio gran parte de los cambios en las lógicas de habitar el lugar para el caso de las nuevas generaciones. La ruptura de la transmisión entre las familias y sobre su propio territorio, es reemplazada por el aprendizaje en las escuelas y colegios, donde los niños y jóvenes de todos los grados absorben contenidos positivistas y eurocéntricos, en los que la ciencia es la verdad absoluta, invisibilizando y contribuyendo a denigrar la riqueza de la mentalidad campesina, empírica y profundamente integral, puesto que esta no es competitiva, innovadora, ni tecnológica. 


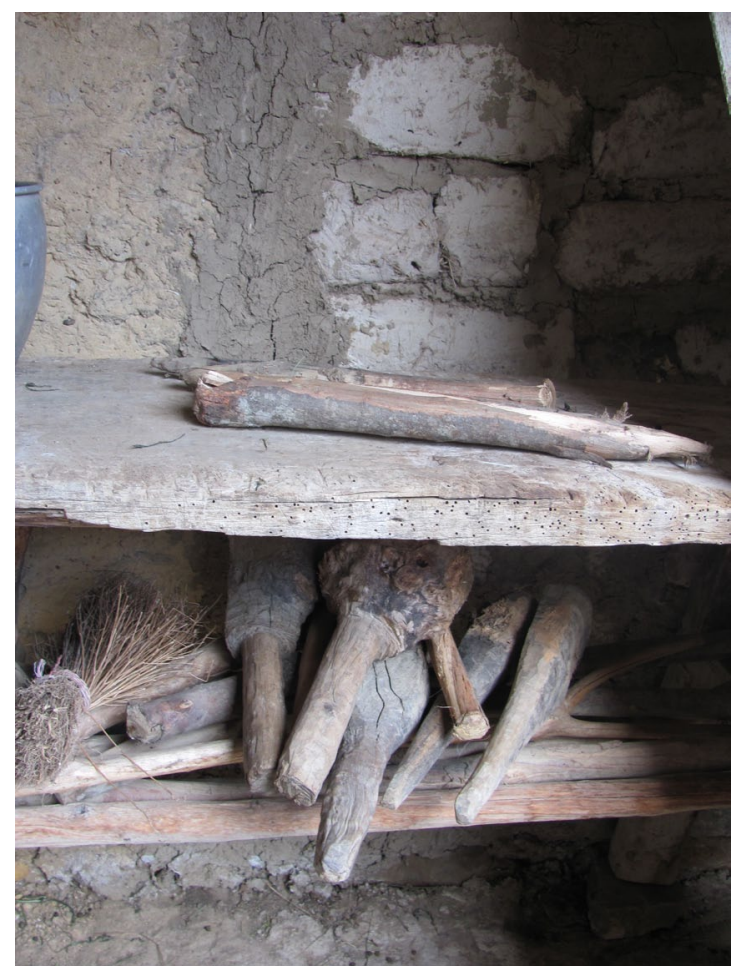

Figura 8. Herramientas en madera propiedad de Hildo López Vereda Escalones, municipio de Cucaita, Boyacá, Colombia. Fuente: Carlos Carreño (2015)

No en vano López (1989) invita a que los procesos educativos rurales en Boyacá sean repensados:

el problema es identificar como se están generando esos cambios: ¿Permiten verdaderamente un mejoramiento de las condiciones de vida?, ¿Lo están logrando con una integración de valores y una afirmación de su propio sentido de pertenencia? o por el contrario ¿Lo que estamos haciendo es generar en la población nuevos valores que llevarán a un rechazo absoluto de sus pautas de comportamiento tradicionales, cotidianas. (López, 1989, pp.149-150)

La ausencia de un sistema educativo integral, consecuente con su entorno, y basado en la necesidad de generar en los niños y jóvenes una consciencia de realidad, producto de un pensar educativo como debate constante entre el profesorado, ha hecho que en Cucaita, como en los otros 122 municipios de Boyacá, se hagan esfuerzos puntuales, sin mayor 
trascendencia ni significación; los docentes, normalmente del área de sociales o artística, para visibilizar aspectos materiales de la tradición boyacense, los convierten en estereotipos o disfraces tales como la ruana o las alpargatas, que se exhiben en carteleras y danzas en días de celebración como el de la Boyacensidad ${ }^{2}$.

Sin embargo, el problema no se limita solo a temas formales y materiales como el pretendido "vestuario típico", para que lo visibilicen desde Puerto Boyacá hasta Tununguá, sino que se asocia y a la vez hace evidente las lógicas ajenas a las realidades locales y diversas del territorio colombiano, como factores de cambio, en este caso, desde el sistema educativo, por ello “...observamos las manifestaciones de estos cambios en la mentalidad colectiva, encontramos que es sorprendente el proceso de amnesia -no de ignorancia- que sufren las generaciones de jóvenes: es un proceso consciente y constante" (López, 1989, p. 150).

\section{Hilando y trenzando el territorio:}

Teniendo en cuenta lo anterior, el Museo de Cucaita sería uno de los referentes de un nodo de la ya mencionada red, a través del cual se podría aportar a la reintegración territorial, no intentando retroceder el tiempo, ni implantando tradiciones hoy extintas, sino abriéndose como espacio reflexivo para el encuentro de diálogos sensibles entre los habitantes, y desde allí, poder trenzar de nuevo, hilar relaciones, facilitar comunicaciones, restituir el sentido colectivo del ser cucaitense hoy: “...nosotros los del campo pienso que si somos libres, es una forma de vida natural, un privilegio virgencita linda..." (M. Sierra, comunicación personal, 12 de noviembre de 2016).

Pero esa función no la puede cumplir tal cual como se encuentra planteado actualmente, es decir, como un espacio cerrado donde se exhiben objetos antiguos. Por ello es necesario pensar de una manera diferente para que se visibilice la realidad activa del territorio, así como debe hacerse él mismo visible como eje dinamizador de sentido y espacio para la participación activa de las comunidades cucaitenses.

Para construir una narrativa coherente con esa realidad activa del territorio es necesario pensar en un contenido que estudie y articule los elementos contemporáneos rurales, no como elementos ajenos o complementarios, sino entendidos como parte integrante y actuante desde

\footnotetext{
${ }^{2}$ Día de celebración institucionalizado desde el año 2009 por la Gobernación de Boyacá, en el que el 2 de octubre de cada año se promueva y fortalezca la "boyacensidad" a través de eventos culturales y folclóricos.
} 
los sistemas o las lógicas económicas, sociales y culturales. Es precisamente en esos elementos donde se pueden observar los sentidos que han tomado los recursos provenientes de las tradiciones y costumbres cucaitenses y las consecuencias de los cambios que han sufrido, en términos de continuidades, resistencias y nuevas inserciones en las lógicas productivas locales (López, 1989).

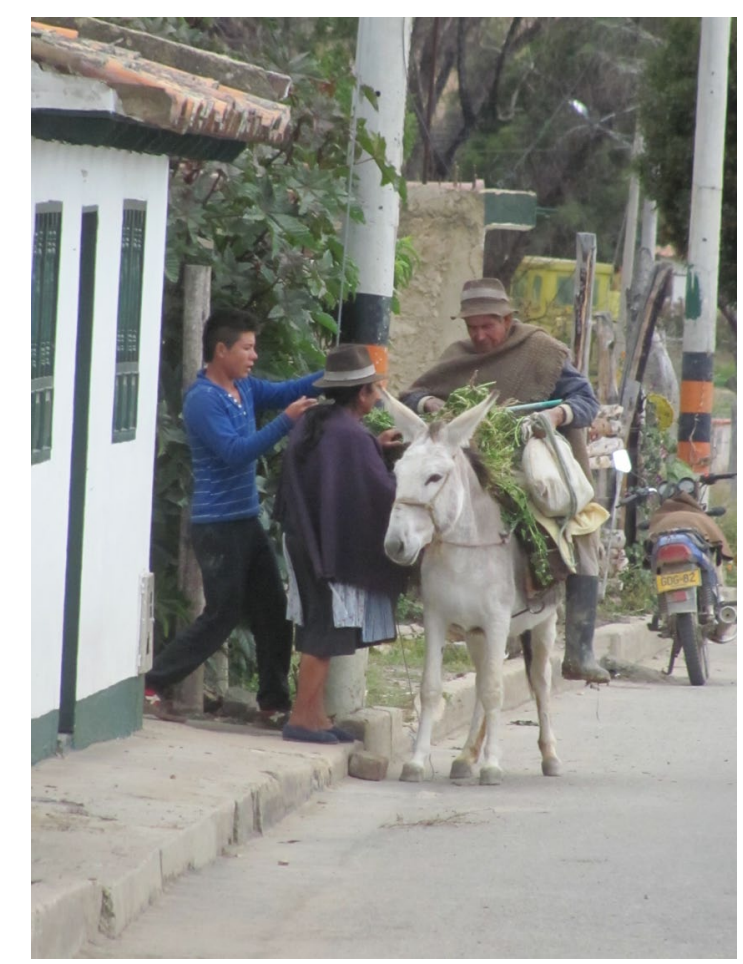

Figura 9. Marco Antonio Sierra Bastidas es recibido por su esposa y su nieto Centro urbano del municipio de Cucaita, Boyacá, Colombia. Fuente: Carlos Carreño 2015

Es en esa relación de causas y consecuencias que se evidencian las tensiones que aportan a la construcción del posible nuevo contenido del museo o, mejor aún, son el pretexto para su aparición en la cotidianidad del municipio, con toda la complejidad que esto conlleva. Hay que dejar de hablar desde el cómodo emplazamiento de objetos en un espacio, esperando a que sus valores formales inciten la curiosidad de quienes los observan, sino de una tarea de expansión de la capacidad comunicativa de este referente, para que dialogue con las comunidades de manera coherente con sus formas y espacios habituales. Se trata de convertir al museo en un 
espacio que permita pensar en la realidad rural cucaitense, los hogares, las escuelas, los sembrados.

Además a los niños de las escuelas rurales nunca los traen por acá y ellos también son habitantes de este lugar. Sabe que sí, eso le cambiaría, que sea un museo para todos los habitantes, no solo los del pueblo. (A. Gil, comunicación personal, 13 de agosto de 2015)

En este punto, se insiste en la importancia de pensar cómo reinventar el museo como ente dialogante, deconstruyéndolo como espacio expositivo para entrar en el ámbito de lo interpretativo y participativo, conscientes de la necesidad de su fortalecimiento como referente cohesionador de esa red ya mencionada que configura las dinámicas morfológicas del territorio.
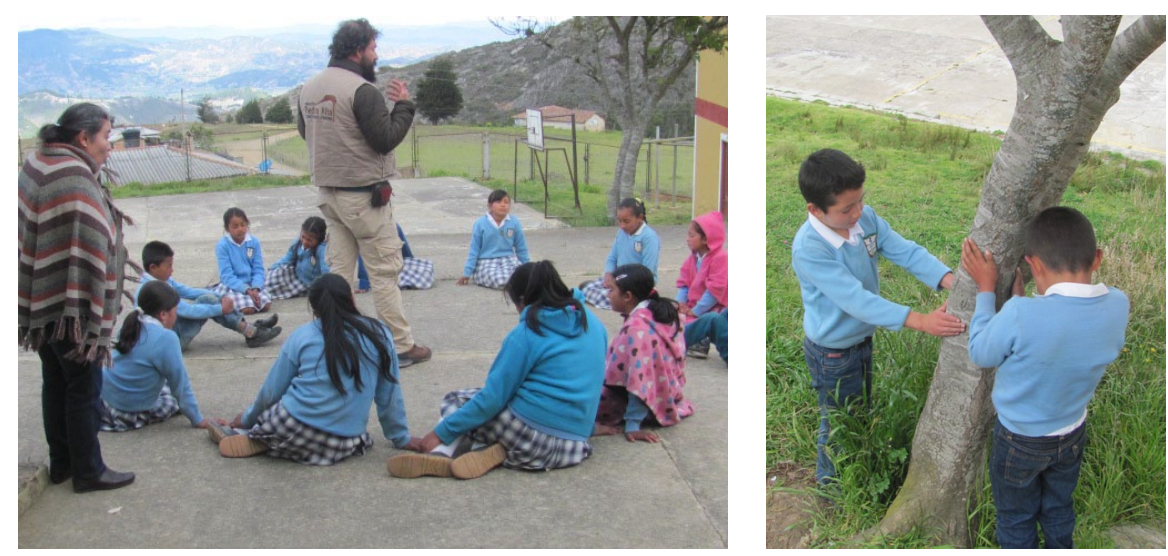

Figuras 10, 11. Momentos de espacios de experiencia sensible para la interpretaciòn del territorio.

Escuela Escalones. Vereda Escalones, Municipio de Cucaita, Boyacà, Colombia. Fuente: Carlos Carreño (2015).

El problema a resolver no es cómo organizar los objetos, sino cómo hacer de su contenido una narración coherente con las lógicas rurales de Cucaita "Pienso yo, que ese museo debería ser como más cercano a nosotros los campesinos...me parece que algo parecido a la huerta de acá atrás..." (I. Parra, comunicación personal, 19 de noviembre de 2015).

Para convertir el museo, no es suficiente una sede física en el centro urbano con un letrero que lo anuncie, toda vez que (de los 4687 habitantes del municipio) el $90 \%$ de las comunidades que habitan el territorio de Cucaita lo hacen en las zonas rurales (4218 habitantes). 
Lo anterior conduce a pensar en estrategias que, primero que todo, desmaterialicen su acervo; para posteriormente diseñar lógicas comunicativas que permitan la interacción activa de los cucaitenses con ese acervo. Deben ser lógicas comunicativas que se adecuen a los espacios de interacción rural, siendo conscientes de que dichos contextos sociales son grupos humanos “...donde ese capital cultural está fundamentado básicamente en una tradición expresada por conglomerados humanos que responden a unas expectativas mínimas de integración de las comunidades y un sentido de pertenencia..." (López, 1989, p. 145).

En este sentido, el museo debe constituirse, no solo como referente visible al interior de un nodo de la red territorial, sino también, ser fuerza motora, como parte de esas pulsaciones que transitan a través de los vasos comunicantes que interconectan y dinamizan la cotidianidad activa de Cucaita. La visibilización del museo estará determinada por su capacidad de introducirse en los espacios cotidianos, en las aulas de clase, en las celebraciones y fiestas populares, en las cocinas de los hogares, en los senderos veredales; así, el museo dejará de ser un elemento impuesto, para pasar a ser un espacio de pensamiento sobre el territorio, donde su dinámica estará determinada por los mismos habitantes, quienes son los que dan sentido a todo este proceso “...sería bonito un museo que fuera como la casa de uno, o mejor, como la cocina, porque es acá donde nos reunimos siempre a conversar, a intercambiar..." (H. Otálora, comunicación personal, 10 de octubre de 2015).

A modo de conclusión, se podría mencionar, que este proceso para la transformación de un museo local, como el caso que nos ocupa, requiere de un esfuerzo conjunto, que parta de un diálogo abierto con las comunidades locales, para que en realidad sea parte importante y activa al interior de sus cotidianidades. Para que esto sea una realidad, hace falta un compromiso comunitario e intersectorial, ya que solo de esta manera, se podría llegar a concebir el museo como un lugar común, una solución para la dignificación del universo rural colombiano. 


\section{Referencias bibliográficas:}

Cohen, A. (1912). Historia y memoria colectiva. Recuperado de http://conceptos.sociales. unam.mx/conceptos final/489trabajo.pdf

De Carli, G. (2004). Vigencia de la Nueva Museología en América Latina: conceptos y modelos. Revista ABRA, 24 (33), 55-75.

Halbwachs, M. (2002). Fragmentos de la memoria colectiva. Recuperado de https://ddd.uab. cat/pub/athdig/15788946n2/15788946n2a5.pdf

Le Golf, J. y Nora, (1978). Hacer la historia. Barcelona, España: Laia

Londoño, E. (1989) Historia prehispánica, permanencias y transformaciones en el altiplano cundiboyacense. En Mora, P. (ed.), Historia y cultura populares. Los estudios regionales en Boyacá (pp. 91-96). Tunja, Colombia: Centro de Investigaciones de Cultura Popular ICBA.

López, L. (1989) Historia y tendencias de las culturas populares en Boyacá. En Mora, P. (ed), Historia y cultura populares. Los estudios regionales en Boyacá (pp. 143-151). Tunja, Colombia: Centro de Investigaciones de Cultura Popular ICBA.

Ortiz, M. L. V. (1998). Identidad cultural y territorio: una reflexión en torno a las comunidades trasnacionales entre México y Estados Unidos. Región y Sociedad IX (15), 105-130.

Vázquez Olvera, C. (2008). Estudio introductorio. Revisiones y reflexiones en torno a la función social de los museos. Cuicuilco, 15 (44), 5-14. 